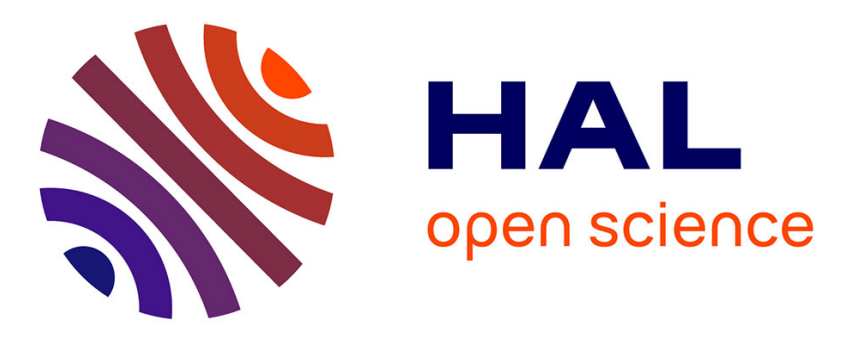

\title{
Novel DSP Receiver Architecture for Multi-Channel Visible Light Communications in Automotive Applications
}

\author{
Alin Cailean, Mihai Dimian, Valentin Popa, Luc Chassagne, Barthélemy \\ Cagneau
}

\section{To cite this version:}

Alin Cailean, Mihai Dimian, Valentin Popa, Luc Chassagne, Barthélemy Cagneau. Novel DSP Receiver Architecture for Multi-Channel Visible Light Communications in Automotive Applications . IEEE Sensors Journal, 2016, 16 (10), 10.1109/JSEN.2016.2529654 · hal-01349085

\section{HAL Id: hal-01349085 \\ https://hal.science/hal-01349085}

Submitted on 17 Aug 2016

HAL is a multi-disciplinary open access archive for the deposit and dissemination of scientific research documents, whether they are published or not. The documents may come from teaching and research institutions in France or abroad, or from public or private research centers.
L'archive ouverte pluridisciplinaire HAL, est destinée au dépôt et à la diffusion de documents scientifiques de niveau recherche, publiés ou non, émanant des établissements d'enseignement et de recherche français ou étrangers, des laboratoires publics ou privés. 


\title{
Novel DSP Receiver Architecture for Multi-Channel Visible Light Communications in Automotive Applications
}

\author{
Alin-Mihai Căilean ${ }^{\dagger}$, Mihai Dimian, Valentin Popa, Luc Chassagne and Barthélemy Cagneau
}

\begin{abstract}
This article presents a novel visible light communications receiver architecture, designed for automotive applications. A central problematic in this area is the design of a suitable receiver able to face the problems caused by the dynamic situations, by the long distances and also by the environmental conditions. In such circumstances, a solution would be to adapt the communication's data rate to channel conditions, meaning that the communication would take place using different data rates depending on the signal to noise ratio (SNR) and the message priority. Considering the digital filtering better performances, the proposed architecture considers the usage of digital signal processing (DSP) as an alternative to the analog signal treatment. The visible light communication receiver proposed in this article addresses the upper mentioned issues and enables a robust communication even at low SNR.
\end{abstract}

Index Terms - digital signal processing; optical communications; vehicle safety; vehicular communications; visible light communication.

\section{INTRODUCTION}

$\mathrm{V}$ ISIBLE Light Communication (VLC) is an emerging technology which uses the visible light (380 to $780 \mathrm{~nm}$ ) produced by LEDs not just for illumination or signaling purposes but also to enable wireless communication. The data transmission capability is achieved by modulating the data onto the instantaneous power of the light, at speeds much faster that the human eye can perceive. Due to the simplicity of the technology, any LED light source can be enhanced with data transmitting capabilities and can become a VLC emitter.

The infrastructure used in this work was partially supported from the project "Integrated Center for research, development and innovation in Advanced Materials, Nanotechnologies, and Distributed Systems for fabrication and control", Contract No. 671/09.04.2015, Sectoral Operational Program for Increase of the Economic Competitiveness co-funded from the European Regional Development Fund.

A.-M. Cailean ${ }^{\dagger}$ and M. Dimian are with the Integrated Center for research, development and innovation in Advanced Materials, Nanotechnologies, and Distributed Systems for fabrication and control, Stefan cel Mare University of Suceava, 13 Universitatii str., 720229, Suceava, Romania, (e-mail: alinc@eed.usv.ro; dimian@usm.ro). (Corresponding author: Alin-Mihai Căilean.).

Valentin Popa is with the Department of Computers, Electronics and Automation, Stefan cel Mare University, Suceava, Romania (e-mail: valentin@eed.usv.ro).

Barthélemy Cagneau and Luc Chassagne are with the LISV laboratory of the University of Versailles Saint-Quentin, 10-12 avenue de l'Europe, 78140 Velizy, France (e-mail: \{luc.chassagne, barthelemy.cagneau\}@ uvsq.fr).
Besides the advantage of being ubiquitous, VLC has plenty other benefits. One of them is the huge available bandwidth which enables VLC to achieve very high data rates as in [1]. The great potential of VLC has been also confirmed with its standardization by the IEEE 802.15.7 standard [2].

Besides the high date rate indoor applications, VLC is also considered suitable for relatively long range outdoor applications, as the vehicle safety communications [3]. Vehicular communications represent a major challenge for the researchers in the transportation field, as it has the potential to significantly reduce the number of traffic accidents [4] and also to increase the efficiency of the transportation system. Furthermore, in the context of an increasing number of road fatalities, the United Nations has declared in 2010 a Decade of Action for Road Safety with the purpose of improving the safety of vehicles and roads, proving again the importance of transportation safety.

In the vehicular communications domain, VLC is able to support both Infrastructure to Vehicle (I2V/V2I) and Vehicle to Vehicle (V2V) communications, as demonstrated in [5] [8], respectively in [9] - [11]. Inter-Vehicle Communication (IVC) enable the vehicles to communicate to each other and to exchange data concerning their state (e.g. position, velocity, engine state, etc.) or information about the traffic conditions (e.g. speed limits, line works, alternative routes, etc.). Besides, IVC have the potential to improve the passenger's comfort.

The usage of VLC in vehicular communications comes as an alternative and/or as a complement for the $5.9 \mathrm{GHz}$ Dedicated Short Range Communications (DSRC). Even though it has unquestionable benefits, DSRC also has several unsolved issues which make it unable to satisfy the requirements imposed for vehicular safety applications [12]. Most of the problems are encountered in the high traffic density scenarios, where the numerous nodes are causing mutual interferences that increase the delays and decrease the packet delivery ratio [13]. In transportation, VLC is favored by the integration of the LED lighting technology in vehicles, traffic lights, traffic sings and street lighting. This fact significantly reduces the implementation cost and facilitates large scale implementation.

The VLC receivers are used to extract the data from the modulated light beam. Concerning the ones used in automotive applications, they are based either on high speed camera systems either on photosensitive elements. The ones 
based on high speed camera systems may offer certain advantages but the increased cost makes them rather unsuitable for the automotive domain. The receivers based on photosensitive elements are significantly cheaper and are also quite efficient in noise performances. Silicon PIN photodiodes, used in a reversed bias configuration offer fast response times (few ns) and have a linear response. The produced photocurrent is processed by a transimpedance circuit and transformed into a voltage that will be further processed until the data signal is reconstructed. The transimpedance circuit has as advantages the low distortion and the large gain-bandwidth product. Therefore, it represents the best compromise between bandwidth and noise [8], [14].

This paper presents a novel VLC receiver architecture designed for mobile multi-channel communications. Unlike in other works [5] - [11], the proposed model uses exclusively digital signal processing techniques in order to process the output of the transimpedance circuit. This approach seems to be better suited for long distance communications where the SNR is low. Furthermore, the flexibility of the digital filters can easily enable the system usage in Multi Input Multi Output (MIMO) applications. The article analyses through numerous simulation the architecture suitability for VLC. The influences of the noise, of the modulation frequency and of the mobile conditions on the Bit Error Ratio (BER) are investigated as well. As far as we know, this is the first paper that proposes such an approach in order to develop VLC receivers. The proposed approach has the potential to open a new path in the development of long distance, low SNR, MIMO VLC receivers for automotive applications. The rest of this article is structured as follows. Section II aims to point out the complexity and the unpredictability of the vehicular VLC channel. Section III describes the implementation of the proposed VLC architecture and explains how it addresses these specific problems. Section IV presents the simulation results, whereas section $\mathrm{V}$ presents the conclusions of this work.

\section{The Influence OF The OUtDoor Channel On VLC PERFORMANCES}

In indoor applications, the emitter - receiver distances are relatively short, in most cases being equal to the distance between ceiling and workspace, as in [15]. On the other hand, in automotive applications, the involved distances are significantly greater, going up to several dozens of meters. Furthermore, due to the dynamic conditions involved by the traffic, the distance is almost continuously changing, and thus the power of the received data signal and the signal to noise ratio (SNR). This makes the VLC channel to be very unpredictable. More problems in outdoor VLC are caused by the weather conditions. The snow, the fog or the rain can cause scattering of the light beam and make the outdoor VLC channel even more unpredictable.

Another major problem in outdoor VLC communications is represented by the other sources of light, besides the VLC emitter. As demonstrated in [16], the outdoor VLC channel is strongly affected by artificial light sources and also by the sun.
If it is too strong, the parasitic light incident on the photosensitive element can saturate the receiver, making it blind and thus block the communication. In order to mitigate the effect of the parasitic light, different solutions such as narrow angle receivers [17], optical filters [18], or different signal processing techniques can be used at the receiver side. Nevertheless, high levels of noise still affect the quality of the communication.

A VLC channel can be modeled as a baseband linear system, with instantaneous power $X(t)$, output photocurrent $Y(t)$ and impulse response $h(t)$ [14]. As suggested in [14], the noise affecting the VLC channel mainly contains a shot noise component and a thermal noise component. The shot noise is proportional to the total optical power incident on the receiver. Even though the effect of the shot noise can be reduced by using optical filters, it still remains a perturbing noise source, limiting the communication's performances. In day-time outdoor communications, the shot noise stands as the dominant noise component. The shot noise problem in automotive applications is very stringent, because in most cases, the power of the parasitic light is much stronger than the one of the light containing the data. The thermal noise is represented by the preamplifier noise and is the predominant noise source in the absence of background light. The shot noise and the thermal noise are characterized as signalindependent and Gaussian noise sources. Therefore, the total noise affecting the VLC channel can be modeled as signalindependent Gaussian noise [14].

\section{IMPLEMENTATION OF THE PROPOSED VLC RECEIVER}

The following section describes the aspects related to the implementation of a novel DSP VLC receiver aimed for automotive applications. It refers to the specific problems in the field and describes how this new receiver architecture addresses them, presenting the structure of the VLC receiver architecture and the structure of the proposed data frame.

\section{A. Considerations on the proposed VLC receiver architecture}

Considering the lower implementation cost, in most of the current outdoor VLC receivers, the output of the transimpedance circuit is treated using analog techniques (see [5 - 11]). Nevertheless, future VLC prototypes could be enhanced by using DSP techniques. The central component of a DSP system is the digital filter. The digital filters can accomplish far better results compared to the analogical ones. As the outdoor VLC channel is strongly deteriorated by noise, the quality of the digital filters is an important advantage which can improve the next generation of outdoor VLC receivers. Considering these aspects, in the proposed receiver (Fig. 1), starting with the output of the transimpedance circuit, the signal processing and reconstruction is achieved using exclusively DSP techniques.

The proposed VLC receiver model aims to be a close replication of a real VLC receiver. It uses the same functionalities and the same working principle. At the same time, it targets to address the challenges imposed by real situations. Basically, the system is meant to be a self-adjusting 


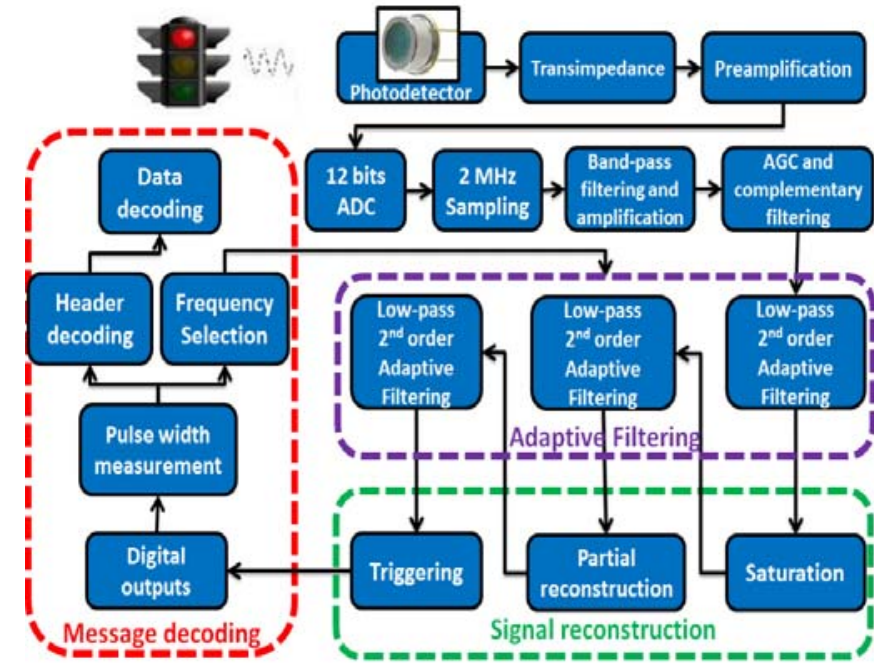

Fig. 1. Architecture of the proposed VLC receiver.

one, able to respond and to adapt to the environment and to different working conditions (such as mobile conditions or variable data rates).

In the considered model, the signal provided by the transimpedance circuit is sampled at a frequency of $2 \mathrm{MHz}$ and at a resolution of $0.008 \mathrm{~V}$, corresponding to a 12 bits ADC resolution for a $3.3 \mathrm{~V}$ input. A higher sampling frequency can significantly improve the quality of the filtering and in consequence the architecture performances. However, considering the future hardware implementation and the cost limitations imposed by the envisioned applications, the designated sampling frequency can be achieved by a moderate priced hardware.

The proposed architecture addresses several key challenges regarding its adaptability. It is well known that in vehicular communication applications, the connectivity and the robustness to noise are more important that the data rate. However, when possible, a higher data rate is desirable. Considering these aspects, the proposed VLC receiver architecture is designed in order to receive and properly decode messages coming at different data rates. This requires an adaptive filtering mechanism able to distinguish between several frequency bands and also able to commute between different frequency bands. This way, the incoming signal containing the data is situated in the filter's band-pass at all time. For the filtering, three $2^{\text {nd }}$ order Butterworth filters have been used, having a cutoff frequency of 1.5 times the modulation frequency. The $2^{\text {nd }}$ order filters have been considered to represent a fair tradeoff between filtering performances and required computation power. In order to be able to properly receive messages coming at different frequencies, the receiver uses an adaptive filtering mechanism. The filters have two inputs: one input is for the input samples (the signal to filter) and the second one is for the cutoff frequency. The second input is connected to the message decoding block, which is continuously commanding the cutoff frequency. As detailed in the next section, the data frame contains a header transmitted at a constant modulation frequency and a data field that can be transmitted using different modulation frequencies. The digital filters adapt their cutoff frequency simply by using a different set of coefficients, corresponding to the new cutoff frequency.

Dynamic conditions, as the ones encountered in traffic situations where the vehicles are in continuous movement, lead to significant variations of the emitter-receiver distance. This phenomenon involves significant variations of the SNR. This problem can be addressed by using an Automatic Gain Control (AGC) mechanism, which maintains a constant signal level and prevents photodetector saturation at short distances, whereas insufficient signal amplification is prevented at long distances. The AGC block helps maintaining a constant signal level required for proper message decoding. It adds a complementary gain to the fixed gain. The complementary gain value is determined after a complex signal analysis. The AGC block ensures a high and steady amplitude level. It considers an optimal signal value, with minimum and maximum thresholds. Whenever the signal's amplitude rises above or falls below the maximum or the minimum thresholds, the new gain value is computed in order to set the signal at the optimal value.

Considering the square pulse reconstruction, it is made based on triggering, according to the values of the thresholds. In order to reduce the pulse width distortions, the pulse reconstruction is a progressive one, which uses a partial reconstruction block before the final triggering. This block uses a multi-level triggering based on an adaptive threshold computing algorithm. Basically, the threshold is continuously changing its value, within certain limits, based on the input samples and on the values of the previous samples. For every pulse, the values of the previous samples are used to determine the signal minimum and the maximum, values that will be used for the threshold computation. Moreover, to help prevent false triggering, the values of the thresholds are modifying their values according to the input signal. The partial reconstruction block enhances the signal by smoothing the lower and the upper part of the signal. The output of this block is a partial square signal which represents an intermediate step towards the final signal reconstruction.

After the square signal has been reconstructed, the message decoding is performed based on pulse width measurement. Based on the width of each pulse and by using a simple decision algorithm, the value of each bit is determined. In the decoding unit, the header is decoded first and based on the information containing the modulation frequency, the cutoff frequency for the data field is selected. Then, the data field is being processed as well.

\section{B. Considerations on the data frame}

A relatively simple digital data frame has been defined. As illustrated in Fig. 2, the structure of the frame consists of a header field and a data field. The header field has a 41 bit length. It begins with a 17 bits preamble used for synchronization. This field enables the receiver to achieve synchronization. It consists of a sequence of zeroes and ones and it can have a variable length. The length of the synchronization is a trade-off between frame overhead and false data acquisitions. 


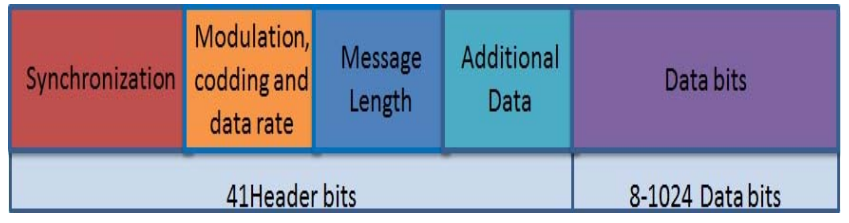

Fig. 2. Structure of the proposed data frame.

The following field of the header is the Modulation and Codding Scheme (MCS) field. The MCS field uses 6 bits to provide the receiver with information regarding the selected modulation, coding technique and also about the data rate. The next field of the header, consisting of 10 bits, is the Message Length (ML) field, which provides the receiver with information concerning the number of bits to be received. The last field of the header consists of 8 bits which are not used for the moment. These bits can be used to provide information about dimming or other type of data that could improve the data decoding process. The header field is transmitted at the lowest frequency, namely $11.67 \mathrm{kHz}$, using On-Off-Keying (OOK) modulation. The preamble filed of the header is transmitted without any line coding, whereas for the other three fields, the Manchester code is used. The data field has a variable length which can extend from 8 to 1024 bits. This field is transmitted at variable frequency, between $11.67 \mathrm{kHz}$ and $100 \mathrm{kHz}$. As in the IEEE 802.15.7 standard, the Manchester code is used as a line coding technique.

Since the purpose of this work is to evaluate the architecture of the proposed VLC receiver and to investigate the effect of noise on the quality of the data transmission and on the BER at the physical layer, the use of error correcting codes has been put aside. However, checksum fields can be easily added for both header and data fields.

\section{SimUlATION RESUlTS AND DISCUSSIONS}

The following section aims to summarize the simulation premises, to present the results and to provide a brief discussion referring to the performances of the proposed VLC receiver architecture.

\section{A. Simulation premises}

The following simulations have been performed using MATLAB/Simulink and consider a direct line of sight (LoS) link between emitter and receiver, necessary for a VLC system to work. Considering the considerations from section II, the channel is subject to signal-independent additive Gaussian noise. Surrounding surfaces can cause unwanted reflections or can scatter the VLC signal, creating multipath effects that can affect the communication. However, it has been demonstrated that the multipath effects do not affect VLC, except in the case of short distances, which are not usually fulfilled under normal traffic conditions [17]. For this reason, non-LoS and multipath signals are not included in this simulation.

In the simulation, the clock of the receiver is not synchronized with phase locked-loop (PLL) for simplicity. The usage of asynchronous transmission is encountered in hardware systems in order to maintain the complexity level and the implementation cost as low as possible. Furthermore, the frequencies involved are low enough and the decoding system has no need of time accuracy. However, in order to improve the data rate and to enable additional functionalities (e.g. distance measurements as in [3]) the future version of the system should use PLL.

In order to evaluate the influence of the mobile conditions, and also to test the efficiency of the AGC stage, simulations have been conducted under the premises of a vehicle approaching the emitter at variable velocities, 30 respectively $50 \mathrm{~km} / \mathrm{h}$

The IEEE 802.15.7 standard specifies for outdoor low data rate applications the usage of OOK modulation with an optical clock of $200 \mathrm{kHz}$ and Manchester coding. The data rates mentioned in this case are 11.67, 24.44, 48.89, 73.3 and 100 $\mathrm{kb} / \mathrm{s}$. In our case, similar data rates are aimed but using directly the envisioned modulation frequency. By selecting this approach, future MIMO applications on different communication frequencies could be enabled. Therefore, the resilience to noise for these five communication frequencies

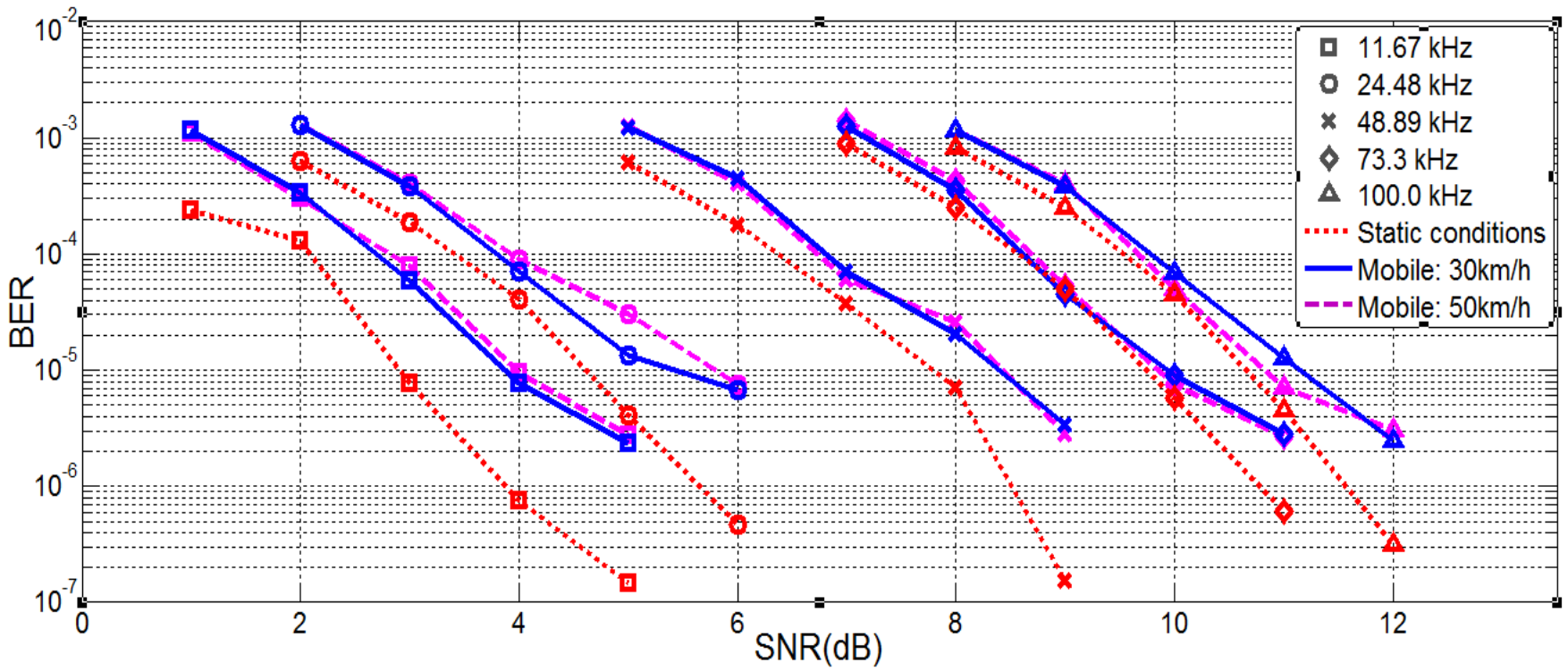

Fig. 3. Bit error ratio for several modulation frequencies. The simulations were performed in static and mobile conditions (at various velocities). 
has been investigated, in order to determine their influence on the communication performances.

\section{B. Simulation results and discussions}

The following section presents the simulation results for the proposed VLC receiver architecture. The purpose of these simulations is to evaluate the performances of the VLC receiver and to determine the influence of noise, modulation frequency and velocity on the BER performances. The BER results for the five modulation frequencies, at different SNRs and velocities are presented in Fig. 3. For the static conditions, the simulations have been undertaken for a BER between $10^{-3}$ (considered adequate for audio transmission) and $10^{-7}$.

The results illustrate the effect of the noise on the receiver's BER results, illustrating the manner in which the SNR decrease determines the BER increases. It can be observed that the BER increase is not as strong in the case of the lower frequencies. The simulation results clearly show that higher frequencies are more sensitive to noise. One of the main reasons for this is the insufficient filtering, which makes the signal reconstruction be lees accurate (fewer samples used for signal processing). As it can be seen, the data rate has a major influence on the BER performances. The results show that for higher data rates, higher levels of SNR are required. Since the signal strength is decreasing as the range is increasing, the low data rates are recommended for long range, whereas as the distance is decreasing (e.g. the vehicle is approaching the traffic light) the data rate can be increased and still maintain the BER within the specified limits. The results also show that at low frequency the proposed VLC receiver architecture is able to maintain the communication and to achieve a decent BER even at low SNRs. Therefore, the low frequencies are suitable for the transmission of the high priority data.

The results also confirm the efficiency of the AGC mechanism and the receiver's suitability in mobile conditions. It can be observed that at lower SNRs, the results for the simulations performed in mobile conditions are quite similar to the ones of the simulations performed under static conditions. Nevertheless, as the SNR increases, the errors are caused mainly by the mobile conditions and less by the noise, creating an increasing gap between the two situations. It can also be observed that in mobile conditions, the BER for the two velocities is very alike. This is because unlike in RF communications, VLC is less sensitive to velocity dependent Doppler spread. Yet, as demonstrated in [7], VLC is affected by the vehicle vibrations, which are velocity dependent. However, because of the absence of a velocity dependent vehicle vibrations model this aspect hasn't been introduced in the simulation.

Regarding the practical implementation of the system, it has as a possible drawback the fact that it requires a relatively powerful processing unit which is able to accomplish the filtering part and data processing in real time. On the other hand, in the case of analog signal processing receivers (see [5]), the microcontroller unit is responsible for fewer operations and low cost processing units can be used. Even though the implementation of such a DSP system may have a higher cost, the digital filter superior performances can enable longer communication distances. Also, the design is simpler since it only uses a processing unit and a frontend transimpedance circuit.

\section{COnClusions}

This article presented a new DSP VLC receiver architecture, optimized for multi-channel vehicular communications and able to work in low SNR conditions. The performances of the proposed receiver were evaluated through simulations. Based upon the simulation results, it was observed the manner in which the noise, the modulation frequency and the vehicle velocity influence the VLC BER performances. The results showed that the proposed system is suitable for the envisioned automotive applications, being able to achieve good BER results even in low SNR conditions. Furthermore, the system multi-channel capability could enable the development of future MIMO applications. Therefore, it can be considered that the usage of DSP techniques can significantly enhance the performances of the VLC receivers, by improving the resilience to noise and also by simplifying the design.

Although the presented VLC receiver wasn't developed based on the IEEE 802.15.7 standard, it should be mentioned that it could be easily adapted for it, once the standard will cover the vehicular communication aspects. Yet, as future work, we plan to adapt the system in order to be standard compliant and to evaluate the impact of standard requirements on the system performances (e.g. BER, throughput, latencies).

\section{REFERENCES}

[1] D. Tsonev, Chun Hyunchae, S. Rajbhandari, J.J.D. McKendry, S. Videv, E. Gu, M. Haji, S. Watson, A.E. Kelly, G. Faulkner, M.D. Dawson, H. Haas, D. O'Brien, "A 3-Gb/s Single-LED OFDM-Based Wireless VLC Link Using a Gallium Nitride $\mu$ LED," Photonics Technology Letters, IEEE, vol.26, no.7, pp.637,640, 2014.

[2] IEEE Standard for Local and Metropolitan Area Networks--Part 15.7: Short-Range Wireless Optical Communication Using Visible Light, IEEE Standard, 2011, 1-309.

[3] S.-H. Yu; O. Shih, H.-M. Tsai; R. Roberts, "Smart automotive lighting for vehicle safety," IEEE Commun. Mag., vol.51, no.12, pp.50,59, 2013.

[4] U.S. Department of Transportation Research and Innovative Technology Administration, Report: Frequency of Target Crashes for IntelliDrive Safety Systems, October 2010.

[5] A.-M. Cailean, B. Cagneau, L. Chassagne, M. Dimian, V. Popa, "Novel Receiver Sensor for Visible Light Communications in Automotive Applications," IEEE Sensors J., vol.15, no.8, pp.4632-4639, 2015.

[6] C. Gavrincea, J. Baranda, P. Henarejos, "Rapid prototyping of standardcompliant visible light communications system," IEEE Commun. Mag., vol.52, no.7, pp.80-87, July 2014.

[7] S. Okada, T. Yendo, T. Yamazato, T. Fujii, M. Tanimoto, Y. Kimura, Y., "On-vehicle receiver for distant visible light road-to-vehicle communication," in Proc. IEEE Intell. Vehicles Symp., June 2009. pp. 1033-1038

[8] N. Kumar, D. Terra, N. Lourenco, L.N. Alves, Rui L. Aguiar, "Visible light communication for intelligent transportation in road safety applications," in Proc. 7th Int. Wireless Commun. and Mobile Computing Conf. 2011, pp.1513,1518.

[9] A. Cailean, B. Cagneau, L. Chassagne, S. Topsu, Y. Alayli, J-M. Blosseville, "Visible light communications: Application to cooperation between vehicles and road infrastructures," in Proc. IEEE Intell. Vehicles Symp. (IV), June 2012, pp.1055,1059.

[10] Deok-Rae Kim, Se-Hoon Yang, Hyun-Seung Kim, Yong-Hwan Son, Sang-Kook Han, "Outdoor Visible Light Communication for inter- 
vehicle communication using Controller Area Network," in Proc. 4th Int. Conf. Commun. and Electronics (ICCE), Aug. 2012, pp.31,34.

[11] I. Takai, T. Harada, M. Andoh, K. Yasutomi, K. Kagawa, S. Kawahito, "Optical Vehicle-to-Vehicle Communication System Using LED Transmitter and Camera Receiver," IEEE Photonics J., vol.6, no.5, pp.7902513, 7902513, Oct. 2014

[12] A.-M. Cailean, B. Cagneau, L. Chassagne, V. Popa, M. Dimian, "A survey on the usage of DSRC and VLC in communication-based vehicle safety applications," in Proc. IEEE 21st Symp. Commun. and Vehicular Technol. in the Benelux (SCVT), Nov. 2014, pp. 69-74.

[13] S. Eichler, "Performance Evaluation of the IEEE 802.11p WAVE Communication Standard", in Proc. IEEE Vehicular Techno. Conf., 2007.

[14] J.M. Kahn, J.R. Barry, "Wireless infrared communications," Proceedings of the IEEE, vol.85, no.2, pp.265,298, Feb 1997.

[15] Y.-K. Cheong; X.W. Ng; W.-Y. Chung, "Hazardless Biomedical Sensing Data Transmission Using VLC," IEEE Sensors J., vol.13, no.9, pp.3347,3348, Sept. 2013.

[16] C. Kaiyun, C. Gang, X. Zhengyuan, R.D. Roberts, "Experimental characterization of traffic light to vehicle VLC link performance," in Proc. IEEE GLOBECOM Workshops (GC Wkshps), pp.808,812, 2011.

[17] C. Liu, B. Sadeghi, E.W. Knightly, "Enabling vehicular visible light communication (V2LC) networks," Proceedings of the Eighth ACM international workshop on Vehicular inter-networking (VANET '11), ACM, New York, NY, USA, 41-50, 2011.

[18] C.C. Chang, Y.J. Su, U. Kurokawa, B. I. Choi, "Interference Rejection Using Filter-Based Sensor Array in VLC Systems," IEEE Sensors J., vol.12, no.5, pp.1025,1032, May 2012.

Alin-Mihai Căilean received a B.S. degree in Electrical Engineering (2009) and a M.S. in Computer and Communication Networks (2011) from the University of Suceava (Romania). He received his PhD (2014) after a joint program between the University of Versailles St. Quentin en Yvelines (France) and the University of Suceava (Romania). Currently, he is researcher at University of Suceava (Romania). His main research area is related to visible light communications, wireless sensors and vehicle safety applications.

Mihai Dimian received his B.S. in Mathematics (1997) and in Physics (2001), as well as a M.S. in Dynamical Systems from the University of Iassy (Romania). He graduated with a Ph.D. in Electrical Engineering (2005) from the University of Maryland, College Park (USA) and performed post-doctoral research at Max Planck Institute, Leipzig (Germany). He is now full professor and vice-Rector at the University of Suceava (Romania). His research interests are focused on fluctuations and noise in nanoelectronics, stochastic aspects of hysteresis, multiscale analysis and modeling.

Valentin Popa received his B.S. degree in Electronics and Telecommunications (1989) as well as the $\mathrm{PhD}$ (1998) from the University of Iassy (Romania). Currently, he is full professor and rector at the University of Suceava (Romania). He is responsible for multiple research grant agreements. His main research areas are: RFID systems, intelligent sensor networks and wireless data transmission systems.

Luc Chassagne received a B.S. in Electrical Engineering (1994) from Supelec (France) and received his Ph.D. (2000) in optoelectronics from the University of Paris XI, Orsay, (France) for his work in the field of atomic frequency standard metrology. He is now Professor and Director of the LISV laboratory. The topics of interest in his research are nanometrology, precision displacements, sensors and AFM instrumentation.

Barthélemy Cagneau received the Ph.D. degree (2008) in mechanical engineering from the University of Pierre \& Marie Curie-Paris 6, Paris (France). After a postdoctoral position in nano-robotics at ISIR (Institut des Systèmes Intelligents et de Robotique, Paris), he became Associate Professor (2009) with the LISV (Laboratoire d'Ingénierie des Systèmes de Versailles,
Versailles). His research interests include force control, adaptive control, and robust bilateral couplings for micro- and nano-robotics. 